\title{
Analgesia in hip fractures. Do fascia-iliac blocks make any difference?
}

\author{
Jacqueline Callear, Ku Shah \\ John Radcliffe Hospital, Oxford
}

\begin{abstract}
Despite recent national advances in the care for the hip fracture patient, significant morbidity and mortality persists. Some of this morbidity is attributable to the analgesia provided in the hospital setting. The National Institute of Health and Care Excellence and the Association of Anaesthetists of Great Britain and Ireland recommend the use of simple oral analgesia including opioids, with fascia-iliac blocks (FIB) used as an adjunct. Literature review reveals a paucity of evidence on this.

The aim of this project was to evaluate the proportion of patients receiving a fascia-iliac block prior to operative intervention. A secondary aim was to evaluate the efficacy of these blocks through analysis of pre and post-operative opioid usage, post-operative delirium, time to bowel opening, and naloxone use.

Patients who received a fascia-iliac block received significantly less post-operative and total analgesia $(p=0.04, p=0.03)$, had lower rates of delirium $(p=0.03)$ and those patients which were discharged directly home had a shorter inpatient stay $(p=0.03)$. No patients who received a fascia-iliac block (FIB) needed naloxone to reverse opioid toxicity, whilst two without fascia-iliac block did. The results of the project eventually led to the introduction of a hip fracture care pathway which incorporates a single shot fascia-iliac block for all patients who are eligible. Within a two year study period, compliance with fascia-iliac blocks improved from $54 \%$ to $90 \%$.
\end{abstract}

Our experience shows a great improvement in compliance with fascia-iliac blocks in the pre-operative period. This work has also underpinned the introduction of a new hip fracture care pathway ultimately to better patient care and outcomes.

\section{Problem}

An ageing population, increased activity level and bone fragility are contributing to the increasing incidence of proximal femoral fractures (PFF).[1] In the UK the current incidence of hip fractures is estimated at 75,000.[2] This equates to a cost to the National Health Service (NHS) of approximately £2 billion.[2] The incidence of hip fracture is predicted to increase to 101,000 by 2020.[1] Falls in the elderly osteoporotic population resulting in hip fracture is the commonest reason for admission to the orthopaedic ward.[2]

Multi-disciplinary care for the hip fracture patient has reduced one month mortality to $8.2 \%$ in recent years but significant morbidity still exists.[3] Analgesia is a key component in the pre-hospital, preoperative and post-operative time periods for the hip fracture patient. Opioids are often required to provide adequate analgesia but can cause nausea, constipation, and confusion (delirium) in the older patient.

\section{Background}

The fascia-iliac block is a relatively new component of the hip fracture analgesia armament. The National Institute of Health and Care Excellence (NICE) recommends the use of simple oral analgesia including opioids, with fascia-iliac blocks used as an adjunct.[2] Small scale studies have suggested superior analgesic effect with pre-operative fascia-iliac blocks. Fascia-iliac blocks provided superior analgesia to intramuscular morphine in a randomised controlled trial of 48 hip fracture patients. [4] The analgesic effects were significant both at rest and 15 degree flexion of the hip. In a different study, 24/30 patients who received a FIB were able to assume a semi-recumbent position pre-operatively due to improved hip flexion which provided comfort whilst waiting for surgical intervention.[5] FIB allows for ease of lateral patient positioning in the anaesthetic room prior to induction of spinal anaesthesia when compared to intravenous alfentanil.[6] Spinal induction was achieved more quickly and patients found this method more acceptable. FIB significantly reduced the incidence of delirium in intermediate risk patients.[7]

The fascia-iliac block is a safe and simple technique that can be administered by junior doctors,[8] A\&E personnel, [9] and specialist pain nurses [10] with training. Knowledge of the local anatomy and use of the landmark technique should almost eliminate the potential for intraneural or intravascular local anaesthetic administration.[11] Single shot fascia-iliac blocks administered in the emergency department by an emergency physician provided significant decrease in pain from 15 minutes - 8 hours post block when compared to standard parenteral medications. Post block analgesic requirements for patients in the FIB group were minimal.[11]

\section{Baseline measurement}

A retrospective audit of available notes from August 2013 was conducted. All hip fracture patients who met the inclusion criteria were included. The inclusion criteria were: radiographically proven hip fracture, age over 65 years, regular analgesia naive, and spinal 
type anaesthesia. $54 \%$ of all hip fracture patients received a fasciailiac block in the pre-operative period. $65 \%$ of these were administered in the Accident and Emergency and 35\% at induction of anaesthesia. The type of anaesthesia was also noted. $72 \%$ of all hip fracture patients received a spinal anaesthetic as recommended by the National Institute of Health and Care Excellence (NICE), whilst the remaining $28 \%$ of patients received a general anaesthetic.

A prospective review of notes between September-December 2013 was conducted. The same study inclusion criteria were used. 41 patients who did receive a single dose fascia-iliac block either in A\&E or during anaesthetic induction versus 41 patients who received spinal anaesthetic alone were included.

The fascia-iliac blocks were given by trained senior doctors in A\&E or senior anaesthetists in theatre. A standardised aseptic protocol was followed. All fascia-iliac blocks consisted of $0.25 \%$ bupivacaine. Patients with body weight exceeding $50 \mathrm{~kg}$ received $40 \mathrm{mls}$ of $0.25 \%$ bupivacaine. Patients with body weight less than $50 \mathrm{~kg}$ received $30 \mathrm{mls}$ of $0.25 \%$ bupivacaine. A standardised administration approach was used: the injection was administered one third of the way along a line drawn between the anterior superior iliac spine and the pubic tubercle between the fascia iliaca and the fascia lata. Only single dose fascia-iliac blocks were included. All fascia-iliac blocks were recorded on a standard proforma.

Analgesic use was divided into two distinct time periods: preoperative and post-operative. The pre-operative time period included analgesia given by the pre-hospital paramedic team, the accident and emergency department, the trauma ward and anaesthetic staff during induction. The post-operative period included the 72 hour period following surgical intervention. Analgesic use was converted into $\mathrm{mg}$ of oral morphine to allow comparison.[12] All patients received regular doses of paracetamol which was not included in the analgesic calculations. No patients received non-steroidal anti-inflammatory medications.

During the inpatient period four patients died; three in the spinal anaesthetic group and one in the fascia-iliac block group. These patients all survived 72 hours post operatively. They were included in all calculations except those relating to duration of inpatient stay.

Patient discharge was either home to their original place of residence or family residence or to a community hospital. Patients who were considered to be in need of further rehabilitation or enhanced care packages were discharged through a community hospital.

Pre-operative analgesia included that given in the pre-hospital period by the ambulance service. In the pre-operative period patients received $23.1 \mathrm{mg}$ versus $19.1 \mathrm{mg}$ oral morphine equivalent in the $\mathrm{SA}$ and $\mathrm{FI}$ groups respectively. This was not statistically significant $(\mathrm{p}=0.37)$. In the 72 hour post-operative period, the SA and $\mathrm{FI}$ groups received $48.5 \mathrm{mg}$ and $31.5 \mathrm{mg}$ of oral morphine equivalent respectively $(\mathrm{p}=0.04)$. The total analgesic requirements for the FI group was significantly lower than the SA group $(p=0.03)$.
Whilst time to bowel opening and change in AMTS was statistically unchanged, there was a $50 \%$ reduction in delirium in the $\mathrm{FI}$ group $(\mathrm{p}=0.03)$. In the $\mathrm{Fl}$ group there were 9 patients $(22 \%)$ with postoperative delirium compared to 18 patients $(44 \%)$ in the spinal anaesthetic group.

Total length of stay in the John Radcliffe Hospital was not significantly different between the two groups. However, for those patients that were being discharged directly home from the ward, the FI group were discharged home sooner $(p=0.03)$.

$67 \%$ of all hip fracture patients during this study period received a fascia-iliac block in the pre-operative period. $71 \%$ of these were administered in the Accident and Emergency and 29\% at induction of anaesthesia. The type of anaesthesia was also noted. $74 \%$ of hip fracture patients received a spinal anaesthetic.

\section{Design}

The early study results suggested significant reduction in opioid use and its' associated morbidity in the patient group receiving a fasciailiac block. The intervention therefore was concentrated on dissemination of these early study results to the practitioners delivering the blocks. Discussions were led at monthly fracture fragility and Accident and Emergency meetings. These meetings included a large number of the members of the multi-disciplinary team: physiotherapists, occupational therapists, pharmacist, physicians, surgeons, ortho-geriatricians, trauma consultants, accident and emergency consultants, and anaesthetists. The results created lively debates and enthusiasm for the ongoing project. It was hoped that the fascia-iliac block could be incorporated into a new hip fracture care pathway.

\section{Strategy}

PDSA cycle 1: Retrospective audit of notes from August 2013 revealed $54 \%$ of patients with hip fracture received a fascia-iliac block. These were either given in A\&E (65\%) or at induction of anaesthesia (35\%). $72 \%$ of hip fracture patients received spinal type anaesthetic. The remainder received general anaesthetic (28\%).

PDSA cycle 2: A prospective data collection study was performed between September-December 2013. The efficacy of a single shot fascia-iliac block was evaluated through analysis of: pre and postoperative opioid usage, post-operative delirium, time to bowel opening, naloxone use, and length of hospital stay. This stage of the cycle provided the evidence base to support the routine introduction of a single shot fascia-iliac block to all patients with hip fracture.

PDSA cycle 3: A presentation was given at the monthly fragility fracture meeting to discuss the initial study results. The meeting was attended by physiotherapists, occupational therapists, pharmacists, anaesthetists, senior house officers, registrars, consultant surgeons, and ortho-geriatricians. The importance of fascia-iliac blocks in the hip fracture patient was discussed. 
Everyone felt that the initial results were positive and were keen to engage further with the project.

PDSA cycle 4: A presentation was delivered to the Accident and Emergency team at their departmental governance meeting. Accident and Emergency physicians, practitioners, physiotherapists, and pharmacists were in attendance. They were encouraged to learn that the fascia-iliac blocks given in Accident and Emergency department were having positive impact on the overall outcomes of this patient group. They were keen for more widespread teaching and education about the fascia-iliac block technique, thus enabling more patients to have an anaesthetic block early after entry to the hospital.

PDSA cycle 5: An informal lecture was given was given to the trauma and orthopaedic SHOs. $100 \%$ of orthopaedic juniors were in attendance. The importance of adequate analgesia prescription with regular review was discussed. A pharmacist was present to support the discussion. An admission bundle was devised. This included regular and as required analgesia, regular laxatives, and low molecular weight heparin prescription according to hospital protocol.

PDSA cycle 6: A new standardised hip fracture admission pathway was devised. This was a multi-disciplinary team decision based upon earlier study results. The aim is for every hip fracture patient to receive a single fascia-iliac block on admission to the Accident and Emergency department.

\section{Post-measurement}

PDSA cycle 1: This cycle provided baseline results for the study. $54 \%$ of all hip fracture patients received a fascia-iliac block. These were given in A\&E (65\%) or in the anaesthetic room (35\%). Only $72 \%$ of anaesthesia was spinal type which reflects the recommendations of the National Institute of Health and Care Excellence (NICE).

PDSA cycle 2: Patients who received a fascia-iliac block received significantly less post-operative and total analgesia $(p=0.04$, $p=0.03)$, had lower rates of delirium $(p=0.03)$ and those patients which were discharged directly home had a shorter inpatient stay $(p=0.03)$. There was no use of naloxone to reverse opioid toxicity in the FIB group. The proportion of hip fracture patients receiving a FIB increased from $54 \%$ to $67 \%$. These were either given in A\&E $(71 \%)$ or at induction of anaesthesia (29\%). The use of spinal type anaesthesia increased from $72 \%$ to $74 \%$.

PDSA cycle 6: A new hip fracture fast track protocol was introduced. $90 \%$ of hip fracture patients now receive a single shot fascia-iliac block on admission. Compliance with spinal type anaesthesia has also significantly improved from $62 \%$ at the beginning of the study period to $83 \% .90 \%$ of patients had regular inpatient prescriptions which reflected the admission bundle for analgesia, laxatives and low molecular weight heparin as discussed in PDSA cycle 5.

\section{Lessons and limitations}

This project highlighted the importance of multi-disciplinary working. Analgesia for the hip fracture patient involves a wide number of different healthcare professionals. It is only by collaborative work between all these groups that improvement in patient care can really be achieved. The very strongly positive results gained from PDSA 2 provided the evidence needed to eventually support widespread introduction of the fascia-iliac block to the hip fracture admission pathway.

Multi-disciplinary working is not always straightforward. There can be many different ideas as to how a clinical situation should be improved. It is always important to have a project lead that can liaise between the different specialist groups to develop a unified pattern of thought to solve an identified problem.

\section{Conclusion}

All hospitals admitting patients with proximal femur fractures should have a dedicated care pathway. Each pathway should ultimately incorporate regional anaesthesia like the fascia-iliac block. This project suggests that single dose fascia-iliac blocks given in the preoperative period significantly reduce the post-operative and total analgesic requirements in the hip fracture patient. These patients experience lower rates of delirium and those being discharged directly home do so more quickly. This not only reduces the cost of inpatient stay but also lessens the burden of hip fracture care on elderly comorbid patients.

\section{References}

1 British Orthopaedic Association. The care of patients with fragility fractures. 2007:4.

2 NICE. The management of hip fractures in adults. 2014: 7-9.

3 Royal College of Physicians. Falls and fragility fracture audit programme (FFFAP). National hip fracture database extended report. 2014.

4 Foss N, Kristensen B, Bundgaard M, Bak M, Heiring C, Virkelyst $\mathrm{C}$, et al. Fascia iliaca compartment blockade for acute pain control in hip fracture patients. A randomized, placebo-controlled trial. Anesthesiology. 2007;106:773-778.

5 Candal-Couto JJ, McVie JL, Haslamb N, Innesa AR, Rushmerb J. Pre-operative analgesia for patients with femoral neck fractures using a modified fascia iliaca block technique. Injury. 2005; 36:505-510.

6 Yun MJ, Kim YH, Han MK, Kim JH, Hwang JW, Do SH. Analgesia before a spinal block for femoral neck fracture: fascia iliaca compartment block. Acta Anaesthesiol Scand. 2009;53(10):1282-1287.

7 Mouzopoulos G, Vasiliadis G, Lasanianos N, Nikolaras G, 


\section{BMJ Quality Improvement Reports}

Morakis E, Kaminaris M. Fascia iliaca block prophylaxis for hip fracture patients at risk for delirium: a randomized placebocontrolled study. J Orthop Traumatol. 2009 Sep;10(3):127-133.

8 Hanna L, Gulati A, Graham A. The Role of Fascia lliaca Blocks in Hip Fractures:A Prospective Case-Control Study and Feasibility Assessment of a Junior-Doctor-Delivered Service. ISRN Orthopaedics.2014: 191306.

9 Hogh A. FICB performed by junior registrars as supplement to preoperative analgesia for patients with hip fracture. Strat Trauma Limb Recon. 2008; 2:65-70.

10 Ayodele O. Nurse administered FICB for pre-operative pain relief in adult fractured neck of femur. Acute pain. 2008 Oct: 145-149.

11 Monzon D, Iserson K, Vazquez J. Single fascia iliaca compartment block for post-hip fracture pain relief. J Emerg Med. 2007; 32(3):257-262

12 Evidence.nhs.uk [Internet]. British National Formulary: Pain management with opioids. 2015. Available from: http://www.evidence.nhs.uk/formulary/bnf/current/guidance-on-pres cribing/prescribing-in-palliative-care/pain/pain-management-withopioids

\section{Declaration of interests}

Nothing to declare.

\section{Ethical approval}

This service improvement project was approved by the hospital committee. 